\title{
All optical wavelength conversion by SOA's in a Mach-Zehnder configuration
}

Durhuus, T.; Jørgensen, C.; Mikkelsen, Benny; Pedersen, Rune Johan Skullerud; Stubkjær, Kristian

Published in:

I E E E Photonics Technology Letters

Link to article, DOI:

$10.1109 / 68.265887$

Publication date:

1994

Document Version

Publisher's PDF, also known as Version of record

Link back to DTU Orbit

Citation (APA):

Durhuus, T., Jørgensen, C., Mikkelsen, B., Pedersen, R. J. S., \& Stubkjær, K. (1994). All optical wavelength conversion by SOA's in a Mach-Zehnder configuration. I E E E Photonics Technology Letters, 6(1), 53-55. https://doi.org/10.1109/68.265887

\section{General rights}

Copyright and moral rights for the publications made accessible in the public portal are retained by the authors and/or other copyright owners and it is a condition of accessing publications that users recognise and abide by the legal requirements associated with these rights.

- Users may download and print one copy of any publication from the public portal for the purpose of private study or research.

- You may not further distribute the material or use it for any profit-making activity or commercial gain

- You may freely distribute the URL identifying the publication in the public portal 


\title{
All Optical Wavelength Conversion by SOA's in a Mach-Zehnder Configuration
}

\author{
T. Durhuus, C. Joergensen, B. Mikkelsen, R.J.S. Pedersen, and K.E. Stubkjaer
}

\begin{abstract}
Penalty free wavelength conversion is demonstrated at $2.5 \mathrm{Gbit} / \mathrm{s}$ over a wavelength span of $12 \mathrm{~nm}$ by the use of SOA's in a Mach-Zehnder configuration. An increase in the extinction ratio is measured for the converted signal compared to the input signal implying signal regeneration as well as wavelength conversion.
\end{abstract}

\section{INTRODUCTION}

$\mathbf{F}$ UTURE optical networks are likely to use the wavelength dimension in order to attain a high transmission capacity at moderate bit rates. In addition to a large capacity the wavelength division multiplexed networks (WDM) offer the possibility of: simple channel routing [1], add/drop functions at low bit rate compared to the transmission capacity [2], and a longer transmission span due to an increased dispersion limit. In such networks wavelength converters, that transform information from one wavelength to another, will be key components [4]-[8], [10]. The role of the wavelength converter can be to act as a wavelength slot interchanger; to direct signals to a given node in the network; or simply to increase the capacity and the flexibility of the network by re-using the available wavelengths [3].

Several techniques have been proposed in the literature to perform the wavelength conversion function, e.g.: i) crosstalk and four wave mixing in semiconductor optical amplifiers [4], [5] ii) gain saturation in DBR lasers [6] as well as iii) injection locking in Y-lasers [7]. An important feature of the wavelength converter is the ability to regenerate or improve the signal extinction ratio over a wide wavelength range. However, for the above mentioned converters this is only possible at relatively high input power levels $(\sim 0 \mathrm{dBm})$.

Here we demonstrate a new type of wavelength converter relying on optically controlled refractive index change in semiconductor optical amplifiers (SOA's). The SOA's are situated in a Mach-Zehnder configuration in order to transfer the phase modulation into an amplitude modulated signal. Using this technique we have demonstrated penalty free wavelength conversion over $12 \mathrm{~nm}$ at $2.5 \mathrm{Gbit} / \mathrm{s}$. The advantage of this concept is the low operation power needed $(<-10 \mathrm{dBm})$ as well as the ability to improve the signal quality of the converted signal with respect to extinction ratio and chirp.

Manuscript received July 21, 1993; revised October 12, 1993.

The authors are with the Center for Broadband Telecommunication, Electromagnetics Institute, Technical University of Denmark, DK-2800 Lyngby, Denmark.

IEEE Log Number 9214923

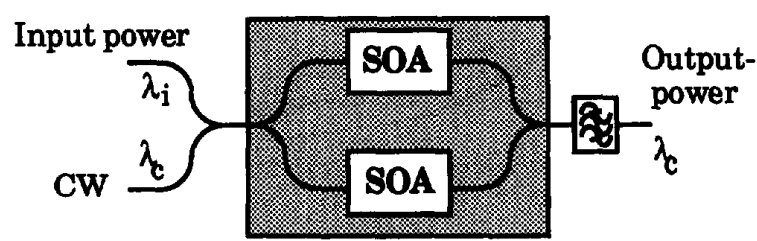

Fig. 1. Schematic of SOA MZI wavelength converter.

\section{PRINCIPLE}

A schematic of the wavelength converter configuration is depicted in Fig. 1. It consists of a Mach-Zehnder interferometer (MZI) with SOA's inserted in the two arms as phase shifting elements. In principle only one SOA is needed [8] but two SOA's give a higher gain. In order to use the configuration for wavelength conversion, asymmetry is required in the MZI, e.g., different splitting ratios in the couplers or asymmetric biasing of the SOA's. The operation is simple: $\mathrm{CW}$ light is injected into the MZI at the wavelength $\lambda_{c}$ and at the output of the converter it will experience constructive or destructive interference depending on the phase shift through the SOA's. The SOA phase shift relies on the change in carrier density that can be controlled via the bias current or the optical input power (gain saturation). If optical power is injected into the MZI at $\lambda_{i}$ the carrier density, N, in the SOA's will change due to the increased stimulated emission. Consequently, the phase, $\Phi$, and thereby the output power at $\lambda_{c}$ will change. So it is possible to vary the output power at $\lambda_{c}$ by varying the input power at $\lambda_{i}$; consequently, wavelength conversion is achieved.

Assuming a true travelling wave amplifier the small signal phase change with photon density is:

$$
\Delta \Phi=-\frac{2 \pi}{\lambda} \frac{\partial n}{\partial N} L \frac{\tau_{e}}{\sqrt{1+\left(\omega \tau_{e}\right)^{2}}} v_{g} g \Delta S \cos (\omega t-\phi)
$$

where $\lambda$ is the signal wavelength, $\frac{\partial n}{\partial N}$ is the refractive index change with carrier density, $L$ the cavity length, $\tau_{e}$ the effective carrier lifetime, $\omega$ is the angular modulation frequency, $v_{g}$ the group velocity, $g$ the material gain, $\Delta S$ the amplitude of the photon density variation and $\phi$ the phase lag between the optical modulation and the carrier density modulation. From this expression it is clear that the phase change depends on the photon density change and thus on the envelope of the incoming signal. As a result, a highly chirped input signal will be converted to a nearly unchirped signal, but with the same envelope. Equation (1) also gives the connection between the conversion speed and the effective carrier lifetime, indicating that highest bit rates are reached with a short carrier lifetime. 


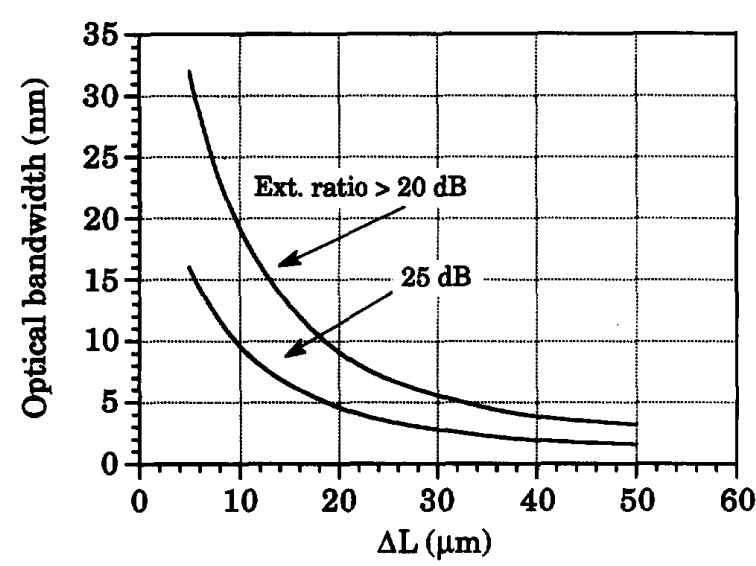

Fig. 2. The optical bandwidth as a function of the MZI arm length difference The parameter is the static extinction ratio.

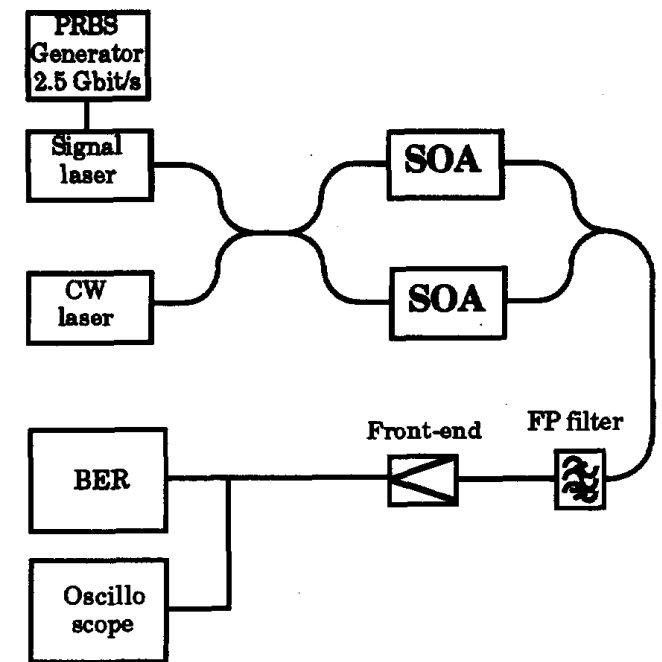

Fig. 3. Experimental set-up for measuring extinction ratio of the converted signal and BER measurements.

In order to attain long term stable operation and large optical bandwidth (small arm length difference) the SOA's and the MZI should be monolithically integrated. The relation between optical bandwidth and MZI arm length difference is shown in Fig. 2 with the static extinction ratio as a parameter. For a optical bandwidth of $20 \mathrm{~nm}$ the arm length difference should be kept below $10 \mu \mathrm{m}$ ( $20 \mathrm{~dB}$ ext. ratio) which is easy to fulfil with a integrated device.

To avoid significant gain changes for a given change in the refractive index the MZI converter should be operating at wavelengths above the gain peak of the SOA's.

\section{EXPERIMENTS}

For a proof of concept the MZI converter was constructed by discrete SOA's and 50\% fiber couplers. Both SOA's have laterally tapered wave guides and buried facets [9] and they

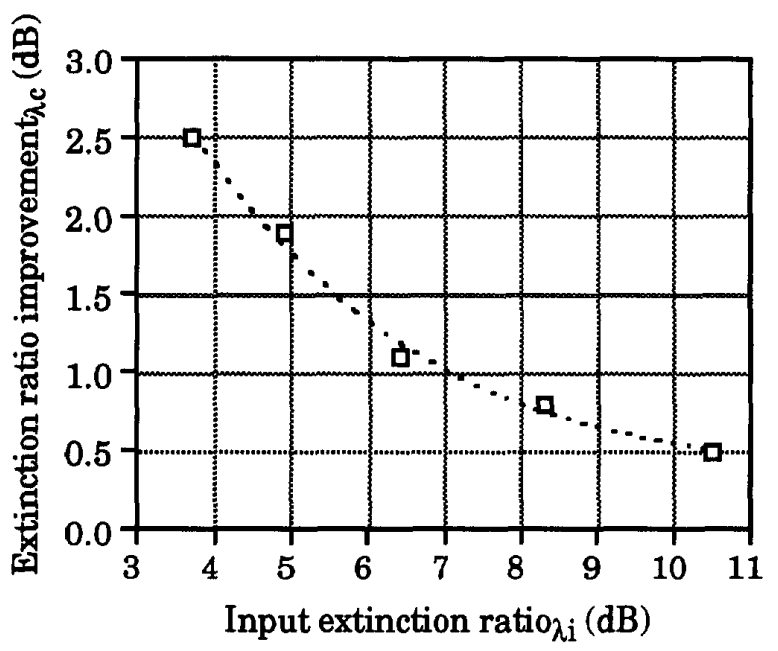

Fig. 4. Extinction ratio improvement for converted signal $(1543 \mathrm{~nm}) \mathrm{com}-$ pared to the input signal $(1531 \mathrm{~nm})$ vs. the input extinction ratio. Bitrate: 2.5 Gbit/s. Signal power: $-11 \mathrm{dBm}$. CW power: $-20 \mathrm{dBm}$.

exhibit a spectral gain ripple and polarization sensitivity below $1 \mathrm{~dB}$. The MZI arm lengths are $10 \mathrm{~cm}$ with a difference below $1 \mathrm{~mm}$ giving a free spectral range (FSR) larger than $200 \mathrm{GHz}$.

To assess the performance the set-up shown in Fig. 3 is used. The CW laser and the signal laser, emitting at 1543 and $1531 \mathrm{~nm}$, respectively, result in coupled powers of -20 and $-11 \mathrm{dBm}$ (avg. $2^{7}-1 \mathrm{PRBS}$ ), respectively. At the output of the MZI the converted signal $(1543 \mathrm{~nm})$ is selected by a Fabry-Perot filter (BW: $100 \mathrm{GHz}$ ) before detection. Figure 4 shows the extinction ratio improvement of the converted signal $(1543 \mathrm{~nm})$ compared to the input signal extinction ratio $(1531 \mathrm{~nm})$. It is clear that the converter improves the signal extinction ratio with the largest improvement for small input extinction ratios. This feature of the MZI converter is very attractive since the signal can undergo conversion in several stages without degradation. To investigate the performance of the MZI converter the BER was measured at $2.5 \mathrm{Gbit} / \mathrm{s}$ with a pattern length of $2^{7}-1$. Longer data patterns will not influence the performance of the converter since low frequency thermal effects are avoided due to the optical modulation of the refractive index. Fig. 5 shows the BER as a function of the received power both for the back to back situation $(\square)$, measured directly for the signal laser, and also for the converted signal $(\triangle)$. The penalty for the converted signal compared to the back to back situation is only $0.7 \mathrm{~dB}$ (BER: $10^{-9}$ ). We believe, that this penalty origins from instabilities in the fiber based interferometer and therefore can be avoided by integration of the SOA's and the MZI. For comparison we also measured the BER for the converted signal $(\diamond)$ using a SOA as the converter (without the MZI). With the same optical input power levels a penalty of $2.5 \mathrm{~dB}$ is measured due to a degradation of the extinction ratio. The degradation of the extinction ratio for conversion to long wavelengths is a result of the shift of the gain peak with carrier density. However, this penalty can be reduced by operating at larger input power levels [10]. 


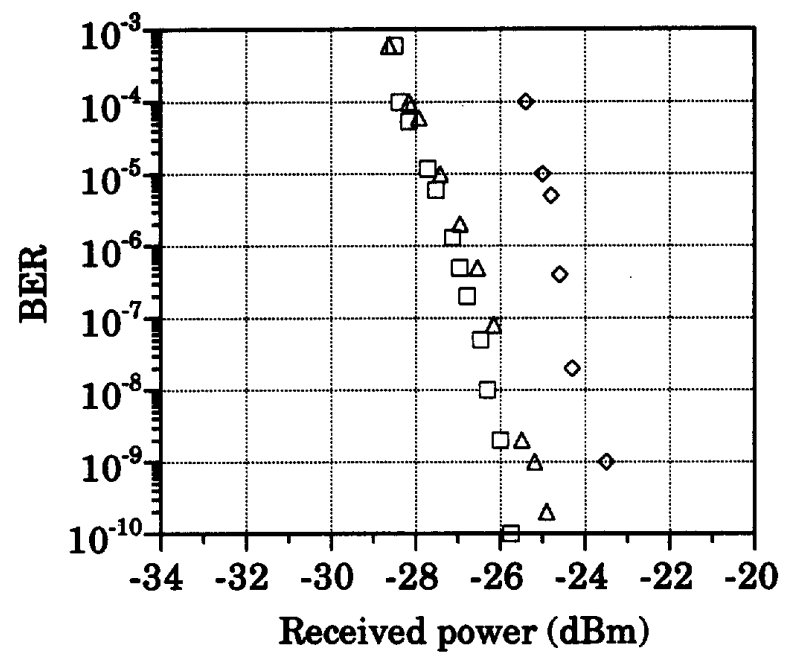

Fig. 5. System experiment at $2.5 \mathrm{Gbit} / \mathrm{s}$. Signal input power: $-11 \mathrm{dBm}$. CW input power: $-20 \mathrm{dBm}$. ( $\square$ ): signal laser (1531 nm) back to back. $(\Delta)$ : Output signal from MZI converter (1543 nm). (o): Signal converted by SOA without MZI configuration (1543 nm).

\section{CONCLUSION}

A new type of wavelength converter based on semiconductor optical amplifiers in a Mach-Zehnder configuration is investigated. Wavelength conversion over $12 \mathrm{~nm}$ is demonstrated at $2.5 \mathrm{Gbit} / \mathrm{s}$. The converter improves the signal quality with respect to extinction ratio while operating at moderate input power levels $(<-10 \mathrm{dBm})$.

\section{ACKNOWLEDGMENT}

Part of this work has been carried out in RACE project 2039. We thank Drs. D. Leclerc, P. Doussire and J. Benoit of Alcatel Alsthom Recherche, Marcoussis for fruitful discussions and for supplying the optical amplifiers.

\section{REFERENCES}

[1] M. Sotom et al., "Photonic network and node architectures for a flexible multigigabit transport network", ICC '93 Workshop, paper 2, Geneva, May 1993.

[2] A. F. Elrefaie, "Multiwavelength survivable ring network architectures". ICC '93, vol. 2, pp. 1245-1251, Geneva, May 1993.

[3] A. A. M. Saleh, "Wavelength division multiplexing", OFC '92, paper ThCl, San Jose, CA, Feb. 1992.

[4] T. Durhuus et al., "High speed all optical gating using two section semiconductor optical amplifier structure", CLEO'92, paper CThS4, Anaheim, CA, May 1992.

[5] G. Grosskopf et al." "140 Mbit/s DPSK transmission using an all- optical frequency converter with a $4000 \mathrm{GHz}$ conversion range", Electron. Lett., vol. 24 , pp. $156-157,1988$.

[6] B. Mikkelsen et al., "Demonstration of all-optical wavelength conversion of high speed signals in coherent and direct detection systems", OFC '93, paper TuH5, San José, CA, Feb. 1993.

[7] M. Schilling et al., "Multifunctional photonic switching operation of $1500 \mathrm{~nm}$ Y-Coupled cavity laser (YCCL) with $28 \mathrm{~nm}$ tuning capability", IEEE Photon. Technol. Lett., vol. 3, pp. 1054-1057, Dec. 1991.

[8] T. Durhuus et al., " $2.5 \mathrm{~Gb} / \mathrm{s}$ optical gating with high on/off ratio by use of SOA's in Mach-Zehnder-Interferometers", CLEO'93, Vol. 11, paper CThH3, Baltimore, MD, May 1993.

[9] P. Doussiere et al., "Polarisation insensitive semiconductor optical amplifier with buried laterally tapered active waveguide", $O A A$ ' 92 paper FA2, Santa Fe, NM, June 1992.

[10] C. Joergensen et al., "Wavelength conversion at $2.5 \mathrm{Gbit} / \mathrm{s}$ using a MachZehnder interferometer with SOA's", OAA '93 paper MD2, Yokohama, Japan, July 1993. 